Martin Bujard

\title{
Einführung in das Schwerpunktthemenheft Elterngeld und Elternzeit in Deutschland: Ziele, Diskurse und Wirkungen
}

Das zum 1. Januar 2007 in Kraft getretene Bundeselterngeld- und Elternzeitgesetz (BEEG) stellt einen Paradigmenwechsel (vgl. Hall 1993) in der deutschen Familienpolitik dar. Zwei neue Charakteristika des Elterngelds kennzeichnen diesen Paradigmenwechsel in besonderer Weise: Erstens ist es eine Lebenslaufpolitik, die einkommensbezogen konzipiert ist, um den Einkommensrückgang in der abgegrenzten Phase nach der Geburt von Kindern auszugleichen. Die Ausgleichsreferenz entspricht daher nicht den klassischen familienpolitischen Prinzipien wie Universalität, Lastenausgleich oder Bedürftigkeit, sondern dem individuellen Lebenslauf - was zu gruppenspezifisch stark unterschiedlichen Leistungshöhen führt. ${ }^{1}$ Zweitens wird mittels der Partnerschafts- bzw. Vätermonate durch einen expliziten Anreiz versucht, in die paarspezifische Aufteilung von Beruf und Fürsorge einzugreifen, um Väter zu einer temporären Erwerbsunterbrechung und zu Fürsorgearbeit zu motivieren. Damit soll die Gleichstellung zwischen Männern und Frauen derart beeinflusst werden, dass sich Männer in dieser Phase etwas an frauentypischen Lebensläufen orientieren anstatt, wie meistens, umgekehrt.

Da das Elterngeld in Deutschland nur wenige Jahre alt ist, sind der politische Prozess (u.a. Henninger/Wahl 2010) und die Wirkungen (u.a. Wrohlich et al. 2012) ein noch junges Forschungsgebiet. Naturgemäß finden sich daher noch Forschungslücken; dies betrifft die Analyse von unterschiedlichen familiensoziologischen, ökonomischen und demografischen Wirkungen ebenso wie die politikwissenschaftliche Forschung zu Zielen, Diskursen und zum Willensbildungsprozess. Dazu kommt, dass das Interesse der Medien an diesem Thema immens ist. Gründe genug, dass sich dieses Schwerpunktthemenheft dem Elterngeld widmet und versucht - sechs Jahre nach Inkrafttreten - eine interdisziplinäre Bilanz zu ziehen.

Ist das Elterngeld wirksam? Welche seiner Ziele erreicht es und welche nicht? Welche Zielsetzungen hat die Politik damit verbunden? Welche politischen Kräfte unterstützen das Elterngeld aufgrund spezifischer Zielsetzungen? Wird es nach der Bundestags-

1 In gewisser Weise lässt es sich sogar als statuserhaltend klassifizieren, also entsprechend dem Prinzip, das den deutschen Wohlfahrtsstaat charakterisiert (vgl. Esping-Andersen 1990). 
wahl in Frage gestellt oder wird es sogar ausgebaut? Die Fragen der Wirksamkeit und des politischen Prozesses hängen sehr eng zusammen. Wissenschaftliche Wirkungsanalysen zum Elterngeld sind hochgradig politisch. Dies fängt bereits bei der Auswahl des Forschungsdesigns an, z.B. bei der Frage, welches Ziel analysiert wird und mit welchem Vergleichsmaßstab. Andererseits können politische oder mediale Akteure partielle Ergebnisse verwenden, um ihre Interessen zu verfolgen. Die Wissenschaft kann instrumentalisiert werden. Insofern ist es wichtig, und das ist das zentrale Anliegen dieses Heftes, eine Brücke zwischen Wirkungsanalysen, Diskursen und politischen Prozessen zu bauen. Politikwissenschaftliche Analysen profitieren von einer tieferen Kenntnis der quantitativen Wirkungsforschung, genauso wie umgekehrt Vertreter dieser Forschungsrichtung von einer Sensibilität gegenüber politischen Zielen und Prozessen profitieren.

Im Folgenden werden beispielhaft zwei Analysen dargestellt - zum einen als Appetizer für diesen Band, zum anderen um zu zeigen, dass je nach Vergleichsmaßstab ambivalente Befunde existieren können. Es werden Veränderungen in Einkommen und Väterbeteiligung analysiert, also in den beiden Bereichen, die den Paradigmenwechsel in Form des einkommensabhängigen Lebenslaufansatzes und des Gleichstellungsimpulses kennzeichnen.

\section{Einkommensveränderungen im Vergleich zwischen Gruppen und im Lebenslauf}

Vergleicht man unterschiedliche sozioökonomische und soziodemografische Gruppen, zeigt sich, dass diese in unterschiedlichem Ausmaß vom Elterngeld profitieren. Tabelle 1 zeigt Mikrozensusauswertungen ${ }^{2}$ des Nettoäquivalenzeinkommens (Haushaltsebene) von Müttern und Vätern mit Babys (= Kindern im Alter von unter einem Jahr) von 2006 im Vergleich zu 2008, also vor und nach Einführung des Elterngelds. ${ }^{3}$ Die Unterschiede zwischen beiden Jahren lassen sich zwar nicht komplett auf das BEEG zurückführen, ein zentraler Einfluss ist jedoch plausibel, da sich der Referenzwert von Männern und Frauen nur um 13 bzw. $16 €$ (ohne Kinder $-6 €$ ) verändert hat; während er in Haushalten mit Babys um durchschnittlich 121 bzw. $113 €$ angestiegen ist. Dieser Anstieg ist hochsignifikant, der t-Wert des Mittelwertvergleichs liegt bei 4,80 bzw. 4,84. Besonders profitiert vom Elterngeld haben Eltern mit hohem Bildungsabschluss, deren Berufseinstieg bereits abgeschlossen ist. Der Anstieg von 2006 auf 2008 ist in Westdeutschland größer als in Ostdeutschland, bei Ehepaaren größer als bei Alleinerziehenden, mit Hochschulabschluss und im öffentlichen Dienst größer und bei Enddreißigern größer als bei jüngeren Eltern.

2 Da das Haushaltseinkommen im Mikrozensus nur kategorial erfasst wird, wird für die Berechnungen der jeweilige Kategoriendurchschnitt verwendet.

3 Werte für 2007 sind wenig aussagekräftig, da sowohl Elterngeld als auch Erziehungsgeld möglich waren. 
Tabelle 1: Nettoäquivalenzeinkommen von Müttern und Vätern mit Babys nach Gruppen

\begin{tabular}{lcccccc}
\hline Nettoäquivalenzeinkommen (€) & \multicolumn{3}{c}{ Männer } & \multicolumn{3}{c}{ Frauen } \\
& $\mathbf{2 0 0 6}$ & $\mathbf{2 0 0 8}$ & $\boldsymbol{\delta}$ '08-'06 $^{\prime}$ & $\mathbf{2 0 0 6}$ & $\mathbf{2 0 0 8}$ & $\boldsymbol{\delta}$ '08-'06 \\
\hline Durchschnitt mit Kindern U1 & 1335 & 1456 & $121^{* * *}$ & 1286 & 1399 & $113^{* * *}$ \\
Westdeutschland & 1358 & 1497 & $139^{* * *}$ & 1314 & 1454 & $140^{* * *}$ \\
Ostdeutschland mit Berlin & 1235 & 1269 & 34 & 1178 & 1178 & 0 \\
Ehepaar & 1378 & 1509 & $131^{* * *}$ & 1377 & 1509 & $132^{* * *}$ \\
Nichteheliche Lebensgemeinschaft & 1138 & 1266 & $128^{* *}$ & 1139 & 1266 & $127^{* *}$ \\
Alleinerziehend & - & - & - & 896 & 879 & -17 \\
ISCED 1 (ohne Abschluss) & 791 & 807 & 16 & 791 & 806 & 15 \\
ISCED 3b (Lehrausbildung) & 1137 & 1200 & $63^{* *}$ & 1224 & 1258 & 34 \\
ISCED 5a (Hochschulabschluss) & 1918 & 2048 & $130^{*}$ & 1812 & 2048 & $236^{* *}$ \\
Öffentlicher Dienst & 1442 & 1687 & $245^{* * *}$ & 1573 & 1800 & $227^{* * *}$ \\
Erwerbstätige ohne öffentl. Dienst & 780 & 785 & 5 & 1071 & 1095 & 24 \\
Westdeutschland 20 bis 24 Jahre & 851 & 985 & $134^{* * *}$ & 950 & 989 & 39 \\
Westdeutschland 35 bis 39 Jahre & 1500 & 1602 & $102^{*}$ & 1587 & 1790 & $203^{* *}$ \\
Referenzwert A: 25- bis 40-Jährige ohne Kinder & 1714 & 1708 & -6 & 1794 & 1788 & -6 \\
Referenzwert B: 25- bis 40-Jährige & 1558 & 1571 & 13 & 1512 & 1528 & $16^{*}$ \\
\hline
\end{tabular}

Anmerkungen: $\boldsymbol{\delta}=$ Veränderung. Signifikanzniveaus des Mittelwertvergleichs (t-Test): $* * *=0,001 ; * *$

$=0,01$ und $^{*}=0,1$. Das Nettoäquivalenzeinkommen (Haushaltsebene) ist nach neuer OECD-Skala berechnet und inflationsbereinigt (Basisjahr 2009). Die Durchschnittswerte beziehen sich auf Frauen und Männer, die mit Kindern unter einem Jahr im Haushalt leben.

Quelle: Mikrozensus SUF 2006 und 2008, eigene Berechnung und Darstellung.

Die Verteilungswirkung des BEEG hat zwei höchst unterschiedliche Effekte: Es verstärkt zum einen gruppenspezifische Einkommensunterschiede. Andererseits wird innerhalb des Lebenslaufs der Rückgang des Haushaltseinkommens nach der Geburt von Kindern gebremst. Dadurch werden nicht nur die Achterbahn des Einkommens im Lebenslauf, sondern auch die erheblichen geschlechtsspezifischen Einkommensdifferenzen bei Paaren mit Babys geglättet. Bezogen auf Frauen zeigt Abbildung 1 den durchschnittlichen Verlauf des Nettoäquivalenzeinkommens im Lebenslauf im Vergleich von 2006 vs. 2008 - also vor und nach Wirkung des Elterngelds auf Frauen, deren jüngstes Kind im Babyalter ist.

Infolge des BEEG ist der drastische Rückgang des Nettoäquivalenzeinkommens nach der Geburt eines Kindes deutlich gebremst: Es fällt von rund $1.790 €$ nicht auf $1.286 €$ wie 2006, sondern es fällt nur auf rund $1.400 €$. Nach Einführung des Elterngelds ist das Einkommen mit Kleinkindern am geringsten. Der t-Test zeigt, dass die Unterschiede zwischen 2006 und 2008 nur beim Alter 0 hochsignifikant (1\%-Niveau) sind. Für einzelne Gruppen von Frauen ist die Glättung des Einkommensrückgangs nach der Geburt noch deutlicher. Die Befunde zeigen, dass das Elterngeld zielgerichtet an der Stelle im Lebenslauf eingreift, bei der die größten Einkommensschwankungen liegen - also v.a. bei den jungen Eltern nach der Geburt der Kinder. 
Abbildung 1: Nettoäquivalenzeinkommen von Frauen nach Alter des jüngsten Kindes

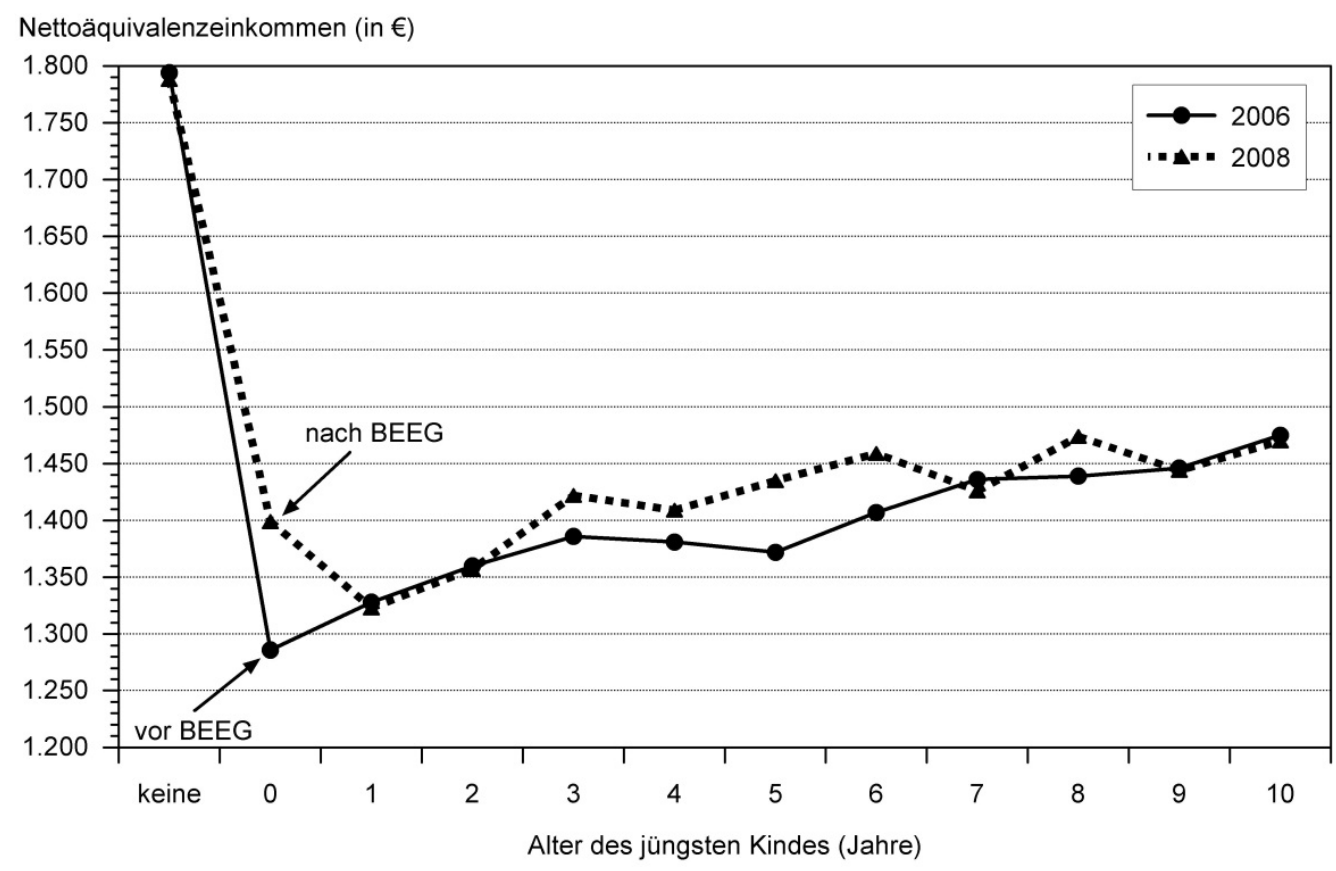

Anmerkungen: Das Nettoäquivalenzeinkommen (Haushaltsebene) ist nach neuer OECD-Skala berechnet und inflationsbereinigt (Basisjahr 2009). Die Durchschnittswerte beziehen sich auf Frauen mit Kindern im Haushalt von 0 bis 10 Jahren, mögliche Effekte des Elterngelds betreffen nur das Alter 0 .

Quelle: Mikrozensus SUF 2006 und 2008, eigene Berechnung und Darstellung.

\section{Väterbeteiligung zwischen steigender Vätermonatsquote und konstanter Arbeitszeit}

Inwieweit das Elterngeld eine verstärkte Väterbeteiligung an der Fürsorge ihrer Babys bewirkt hat, lässt sich an der zunehmenden Nutzung von Elterngeld durch Väter belegen. Abbildung 2 zeigt, dass der Anteil von Vätern mit familienbedingten Erwerbsunterbrechungen, der vor dem BEEG bei 3,5\% $\mathrm{lag}^{4}$, durch das Elterngeld sprunghaft angestiegen ist. Zudem steigt er von Jahr zu Jahr weiter an. Der Bundesländervergleich zeigt, dass die Väterbeteiligung an der Elternzeit nach neuesten Daten ${ }^{5}$ in allen Bundesländern über 16\% liegt, im Bundesdurchschnitt sind dies 25,7\%. In Bayern, Sachsen und Berlin nimmt sogar etwa jeder dritte Vater Elternzeit.

4 Dieser Wert bezieht sich auf den Männeranteil an den Leistungsbeziehern von Erziehungsgeld 2006.

5 Die Daten beziehen sich auf im dritten Quartal 2010 geborene Kinder (vgl. Statistisches Bundesamt 2012). 
Abbildung 2: Zunehmender Elterngeldbezug durch Väter nach Bundesländern

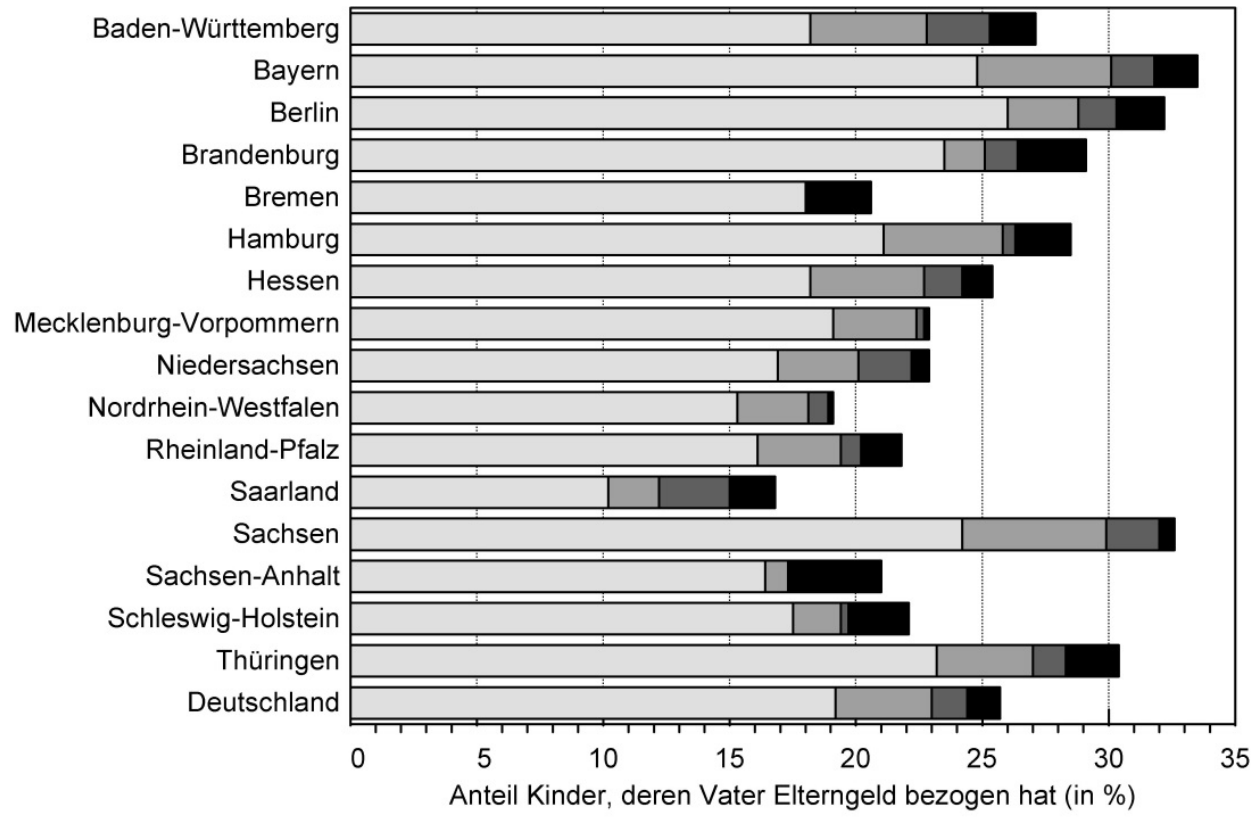

Väterbeteiligung Kinder * 1. Quartal 2008

Väterbeteiligung Kinder * 1. Quartal 2010

Väterbeteiligung Kinder * 1. Quartal 2009

Väterbeteiligung Kinder * 3. Quartal 2010

Quelle: Statistisches Bundesamt 2012, eigene Darstellung.

Diese zunehmende Elterngeldnutzung durch Väter kennzeichnet einen erheblichen Wandel. Allerdings beträgt die durchschnittliche Nutzung nur 3,3 Monate, verglichen mit 11,7 Monaten bei den Müttern. Betrachtet man im Mikrozensus den Anteil von Männern, die mit einem Baby im Haushalt leben, sind zu einem Befragungszeitpunkt X nur knapp 5\% in Elternzeit. Dabei gibt es durchaus einige Gruppen von Männern, die überdurchschnittlich oft Elternzeit nehmen: Beschäftigte im öffentlichen Dienst, Väter von mehreren Kindern, über 35-Jährige, Akademiker und Großstädter (vgl. Geisler/Kreyenfeld 2011).

Bei der wöchentlichen Arbeitszeit von berufstätigen Vätern nach Alter des Kindes für 2005-2009 (siehe Abbildung 3), lässt sich keine Veränderung der Arbeitszeit von Vätern erkennen, die im Zusammenhang mit dem Elterngeld stehen könnte. Die Arbeitszeit ist konstant hoch: unabhängig davon, ob diese vor oder nach der Einführung des Elterngeldes gemessen wird, aber auch unabhängig vom Alter des Kindes. Dagegen wird ein Rückgang der durchschnittlichen Arbeitszeit durch eine andere politische Maßnahme deutlich: Das Kurzarbeitergeld in Folge der Finanzkrise manifestiert sich in einer geringeren Durchschnittsarbeitszeit im Jahr 2009 für Väter von Kindern aller Altersgruppen. 
Abbildung 3: Arbeitszeit von Vätern nach Alter des Kindes 2005-2009

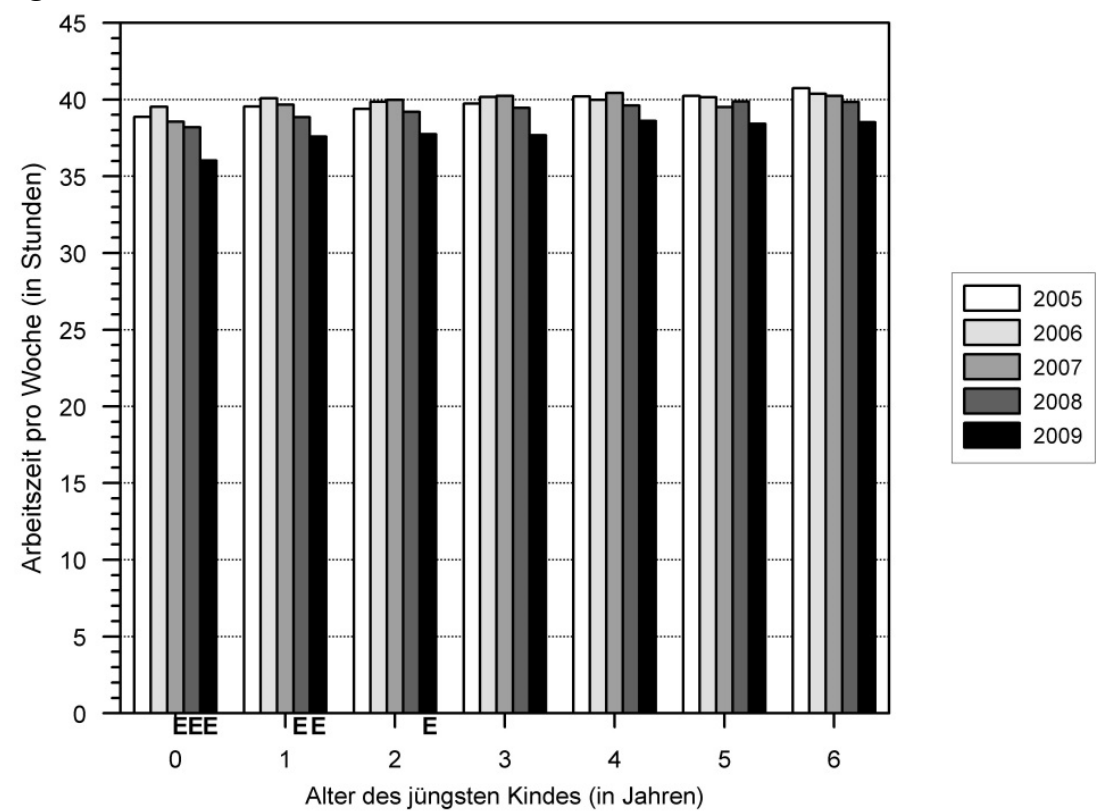

Quelle: Mikrozensen 2005-2009, eigene Berechnung und Darstellung. E = Elterngeld möglich.

Die Abbildungen 2 und 3 machen scheinbar höchst unterschiedliche Aussagen bezüglich der Gleichstellungswirkung des Elterngelds. Wie lassen sich diese - auf den ersten Blick widersprüchlichen - Befunde erklären? Tatsächlich nehmen viele Väter Elternzeit und die Anzahl steigt von Jahr zu Jahr. Das bedeutet, dass ein Teil der heutigen Vätergeneration intensive Erfahrungen mit der Fürsorge von Babys macht ${ }^{6}$ - wertvolle Erfahrungen, die den Generationen vor ihnen nur selten möglich waren. Die langfristigen Folgen dieses Erfahrungsprozesses für Partnerschaften, Vater-Kind-Verhältnisse und Familienleitbilder sind noch nicht abzusehen. Allerdings bleiben Väter trotz dieser temporären Auszeit in der Ernährerrolle. 74,3\% der Väter nehmen keine Elternzeit, die Elternzeit-Väter nehmen nur ein Viertel soviel Monate wie die Frauen und die durchschnittliche Wochenarbeitszeit während der Elternzeit beträgt bei den Männern 15,3 Stunden, während sie bei den Frauen nur bei 1,3 Stunden liegt (Bujard/Schiefer 2012). Diese drei statistischen Effekte verstärken sich gegenseitig, so dass sich die durchschnittliche Gesamtarbeitszeit von Männern kaum verändert.

Für beides, Einkommenseffekte und väterliche Fürsorgezeit, wurden Ambivalenzen der Wirkung aufgezeigt. Durch eine entsprechende einseitige Auswahl aus diesen Befunden hat man empirische Argumente für das Elterngeld - aber genauso auch solche gegen das Elterngeld. Politische Kräfte, die das Elterngeld ablehnen, könnten wie folgt argumentieren:

6 Für die medial verbreitete These, wonach die Vätermonate oft als Urlaub verwendet werden, gibt es keine empirischen Belege. Zumal ein Großteil der Väter direkt nach der Geburt Partnermonate nimmt $(32 \%)$ und eine zweite Häufung dem sequenziellen Muster folgt, bei dem Väter im 13. und 14. Monat Elternzeit nehmen. 
- Beim Nettoäquivalenzeinkommen haben sich die Unterschiede zwischen sozioökonomischen Gruppen verstärkt.

- Die durchschnittliche Arbeitszeit bei Männern ist konstant geblieben, Männer orientieren sich weiterhin an der Ernährerrolle.

Politische Kräfte, die das Elterngeld befürworten, könnten wie folgt argumentieren:

- Im individuellen Lebenslauf hat sich der Einkommensrückgang nach der Geburt von Kindern deutlich reduziert.

- Der Anteil der Väter, die Elternzeit nehmen, hat sich von knapp 5\% mehr als verfünffacht; Väter sammeln in neuartiger Dimension Fürsorgeerfahrungen.

Die Schnittstelle zwischen Wirkungsanalysen und politischer Interpretation ist anfällig für Missverständnisse. Es bedarf expliziter Bewertungen und Einschätzungen nach transparenten Relevanzkriterien, die sowohl von politischer als auch von wissenschaftlicher Seite vorgenommen werden können. Voraussetzung dafür ist beides: ein Überblick über die unterschiedlichen Wirkungen und Wirkmechanismen sowie ein Verständnis der politischen Ziele und Prozesse. Die sechs Beiträge dieses Schwerpunktthemenhefts lassen sich in beide Themenkomplexe einordnen. Drei Beiträge behandeln Ziele, Konzeption und Politikprozess des Elterngelds, drei weitere befassen sich mit Wirkungsanalysen.

\section{Ziele, Diskurse, familienpolitische Konzeption und Politikprozess}

Der Beitrag von Martin Bujard untersucht, welche Ziele in Politik und Medien mit dem Elterngeld verbunden werden und welche Diskurse daraus resultieren. In den empirischen Analysen werden eine Frequenz- und Valenzanalyse von Zeitungsartikeln des Zeitraums 2004-2012 und eine Inhaltsanalyse parlamentarischer Dokumente vorgenommen. Basierend auf diesen Quellen wird das Spannungsfeld von Politik, Medien und Wissenschaft beleuchtet. Der Beitrag zeigt, dass Gesetzgeber und Medien zwar die gleichen fünf Ziele berücksichtigen, die Zielhierarchie sich aber erheblich unterscheidet. Daraus und aus der Valenzanalyse werden Schlussfolgerungen in Hinblick auf die politische Kommunikation und die Einordnung von Wirkungsanalysen zu einzelnen Zielen diskutiert.

Hans Bertram und Carolin Deuflhard untersuchen in ihrem Beitrag das Elterngeld aus einer historisch-sozialpolitischen Perspektive, wobei das Konzept der nachhaltigen Familienpolitik analytischer Bezugspunkt ist. Basierend auf der Strukturierung der Trias Zeit, Geld und Infrastruktur wird die Entwicklung der Familienpolitik in der Bundesrepublik Deutschland seit den 1970er Jahren systematisch dargestellt. Die Autoren zeigen gewisse Kontinuitäten in der Entwicklung zum Elterngeld auf, aber auch die Diskontinuität im Verständnis vom Nachteils- zu einem Leistungsausgleich. Der Beitrag verortet das Elterngeld im Kontext anderer familienpolitischer Maßnahmen. Dies ist insofern relevant für Wirkungsanalysen, weil das Elterngeld nicht nur als singuläre Maßnahme zu betrachten ist, sondern aus einer ganzheitlichen Perspektive im Sinne eines Zusammenspiels mehrerer Maßnahmen.

Der Politikprozess bei der Entstehung des BEEG ist Gegenstand des Beitrags von Tilman Mayer und Wiebke Rösler. Dabei werden Veränderungen der Positionen in den 
Volksparteien SPD und CDU sowie der Einfluss einzelner Akteure gezeigt, die Mitvoraussetzung für die Einführung des Elterngelds durch die große Koalition waren. Die Analyse beschreibt auch Kritik und Unterstützung von Familienverbänden, Arbeitgebern und Kirchen sowie die Rolle wissenschaftlicher Expertisen im politischen Willensbildungsprozess. Abschließend wird ein Exkurs zur Geburtenentwicklung vorgenommen, wobei anhand deskriptiver Statistiken zum Geburtenaufschub Anreize des Elterngelds diskutiert und als Fehlkonstruktion bezeichnet werden.

\section{Wirkungsanalysen des Elterngelds}

Die folgenden drei Beiträge analysieren die Wirkungen des Elterngelds. Johannes Geyer, Peter Haan, C. Katharina Spieß und Katharina Wrohlich analysieren das Elterngeld hinsichtlich seiner Wirkungen auf das Haushaltseinkommen junger Familien und die Erwerbstätigkeit von Müttern. Die Autorinnen und Autoren, die auch an der Evaluation der Wirkungen des BEEG im Auftrag der Bundesregierung beteiligt sind, verwenden ein Mikrosimulationsmodell (STSM), Datengrundlage ist das Sozio-oekonomische Panel (SOEP). Der Beitrag zeigt für die Durchschnittswerte, dass im ersten Lebensjahr das Haushaltseinkommen von Familien gestiegen ist und im zweiten Lebensjahr die Erwerbstätigkeit von Müttern zunimmt. Zudem zeigt das Autorenteam gruppenspezifische Unterschiede der berechneten Effekte.

Martin Bujard und Jasmin Passet gehen der Frage nach, inwieweit sich das Elterngeld auf das Haushaltseinkommen und die Geburtenentwicklung ausgewirkt hat. Dabei werden die differenzierten Einkommensbefunde auch für die Hypothesenbildung der Fertilitätsanalysen verwendet. Empirische Grundlage ist eine Kombination zweier Datensätze: des Mikrozensus für gruppenspezifische Analysen altersspezifischer Geburtenraten und des SOEP für Ereignisanalysen zum Übergang zum zweiten Kind. Der Beitrag zeigt anhand beider Datensätze, dass das Elterngeld gruppenspezifische Fertilitätseffekte aufweist und das Phänomen nachholender Geburten für bestimmte Alters- und Bildungsgruppen verstärkt.

Im letzten Beitrag dieses Schwerpunktthemenhefts analysiert Heike Trappe, inwieweit die Väterzeit als Beschleuniger von Gleichstellung fungiert. Basierend auf Daten von Elterngeldstellen der Bundesländer Bayern, Mecklenburg-Vorpommern und SchleswigHolstein werden Determinanten von Paaren analysiert, bei denen Väter Elternzeit nehmen und bei denen der Elterngeldbezug der Väter länger als zwei Monate ist. Die Befunde werden mit einem Bundesländervergleich von Einstellungen zu Geschlechterrollen, basierend auf ALLBUS-Daten, kombiniert. Trappe zeigt, dass verhandlungstheoretische Erwartungen, aber auch Einstellungen, Wohnort, Parität und der situative Paarkontext eine Rolle bei der Entscheidung spielen können, Vätermonate zu nehmen.

\section{Danksagung}

Für die Erstellung dieses Einführungsartikels dankt der Autor Sabine Gründler, Jasmin Passet, Katrin Schiefer, C. Katharina Spieß und zwei anonymen Gutachterinnen und Gut- 
achtern für hilfreiche Anmerkungen. Für die Entstehung dieses Schwerpunktthemenheftes geht ein großer Dank an alle beteiligten Autoinnen und Autoren, an die Gutachterinnen und Gutachter sowie an Kurt Bierschock.

\section{Literatur}

Bujard, M. \& Schiefer, K. (2012). Arbeitszeit von deutschen Vätern. In: IFP Familienhandbuch. https://www.familienhandbuch.de/?p=14264

Esping-Andersen, G. (1990). The three worlds of welfare capitalism. Cambridge: University Press.

Geisler, E. \& Kreyenfeld, M. (2011): Against all odds. Fathers' use of parental leave in Germany. Journal of European Social Policy 21, 1, S. 88-99.

Hall, P. A. (1993). Policy paradigms, social learning, and the state: The case of economic policymaking in Britain. Comparative Politics 25,3, S. 275-296.

Henninger, A. \& Wahl, A. (2010). Das Umspielen von Veto-Spielern. Wie eine konservative Familienministerin den Familialismus des deutschen Wohlfahrtsstaates unterminiert. In: Egle, C. \& Zohlnhöfer, R. (Hrsg.), Die zweite Große Koalition. Eine Bilanz der Regierung Merkel 2005-2009. Wiesbaden: VS Verlag für Sozialwissenschaften, S. 361-379.

Statistisches Bundesamt (2012). Öffentliche Sozialleistungen. Statistik zum Elterngeld. Beendete Leistungsbezüge für im 3. Vierteljahr 2010 geborene Kinder. Wiesbaden: Statistisches Bundesamt.

Wrohlich, K., Berger, E., Geyer, J., Haan, P., Sengül, D., Spieß, C. K. \& Thiemann, A. (2012). Elterngeldmonitor. Berlin: Deutsches Institut für Wirtschaftsforschung (DIW Politikberatung kompakt $61)$. 